\title{
THE RADIO JET ASSOCIATED WITH THE MULTIPLE V380 ORI SYSTEM
}

\author{
Luis F. Rodríguez ${ }^{1}$, J. Omar Yam ${ }^{1,2}$, Carlos Carrasco-González ${ }^{1}$, Guillem Anglada ${ }^{3}$, and Alfonso Trejo ${ }^{4}$ \\ ${ }^{1}$ Instituto de Radioastronomía y Astrofísica, UNAM, Apdo. Postal 3-72 (Xangari), 58089 Morelia, Michoacán, México; 1.rodriguez@crya.unam.mx \\ ${ }^{2}$ Departamento de Ciencias, Universidad de Quintana Roo, Boulevard Bahía s/n, Col. Del Bosque, 77019 Chetumal, Quintana Roo, México \\ ${ }^{3}$ Instituto de Astrofísica de Andalucía, CSIC, Glorieta de la Astronomía, s/n, E-18008, Granada, Spain \\ ${ }^{4}$ Academia Sinica Institute of Astronomy and Astrophysics, P.O. Box 23-141, Taipei 10617, Taiwan \\ Received 2016 March 30; revised 2016 May 21; accepted 2016 May 23; published 2016 October 3
}

\begin{abstract}
The giant Herbig-Haro object 222 extends over $\sim 6^{\prime}$ in the plane of the sky, with a bow shock morphology. The identification of its exciting source has remained uncertain over the years. A non-thermal radio source located at the core of the shock structure was proposed to be the exciting source. However, Very Large Array studies showed that the radio source has a clear morphology of radio galaxy and a lack of flux variations or proper motions, favoring an extragalactic origin. Recently, an optical-IR study proposed that this giant $\mathrm{HH}$ object is driven by the multiple stellar system V380 Ori, located about $23^{\prime}$ to the SE of HH 222. The exciting sources of HH systems are usually detected as weak free-free emitters at centimeter wavelengths. Here, we report the detection of an elongated radio source associated with the Herbig Be star or with its close infrared companion in the multiple V380 Ori system. This radio source has the characteristics of a thermal radio jet and is aligned with the direction of the giant outflow defined by HH 222 and its suggested counterpart to the SE, HH 1041. We propose that this radio jet traces the origin of the large scale $\mathrm{HH}$ outflow. Assuming that the jet arises from the Herbig Be star, the radio luminosity is a few times smaller than the value expected from the radio-bolometric correlation for radio jets, confirming that this is a more evolved object than those used to establish the correlation.
\end{abstract}

Key words: Herbig-Haro objects - ISM: individual objects (HH 222) - ISM: jets and outflows radio continuum: stars - stars: individual (V380 Ori) - stars: pre-main sequence

\section{INTRODUCTION}

The very large HH object 222 (also known as the Orion streamers) is located in the northern part of the L1641 dark cloud in Orion (Cohen \& Schwartz 1983), near the region where the classic systems HH 1/2 and HH 34 are found. Over the years, different sources have been proposed to excite this shocked region. Reipurth \& Sandell (1985) proposed that a wind from the T Tau star V571 Ori, at only $15^{\prime \prime}$ to the NW of the core of HH 222, was impacting the edge of a cloud and producing the $\mathrm{HH}$ object. A few years later, Yusef-Zadeh et al. (1990) and Morgan et al. (1990) independently detected a strong non-thermal radio source at the core of HH 222 that was proposed as its true exciting source.

Other arguments, however, gravitated against the latter interpretation. Reipurth et al. (1993) did not detect a $1300 \mu \mathrm{m}$ source in association with the non-thermal radio source. The embedded exciting sources of $\mathrm{HH}$ systems typically exhibit detectable radiation at these millimeter wavelengths. Castets et al. (2004) mapped the region around HH 222 in several molecular transitions at $\mathrm{mm}$ wavelengths but failed to detect any dense core that could contain a young driving source. Finally, Trejo \& Rodríguez (2010) obtained new highresolution $6 \mathrm{~cm}$ and $20 \mathrm{~cm}$ continuum images of the radio source and found that it exhibits the double-lobe morphology characteristic of radio galaxies, and that, when comparing data taken with a separation of 17 years, there was no evidence of changes in the flux density and morphology or detectable proper motions at the levels expected for a jet source at the Orion distance. These authors concluded that the non-thermal radio source is most likely a radio galaxy aligned by chance with the line of sight to HH 222. The search for the exciting source of HH 222 was on again.
In Figure 1, we show the $4.86 \mathrm{GHz}$ VLA data for the HH 222 region discussed by Trejo \& Rodríguez (2010). In this image, we have concatenated the 1991 and 2008 data, and selfcalibrated it in phase and amplitude. It is clearly seen from its morphology that the double non-thermal radio source is almost certainly a radio galaxy whose central infrared counterpart is the source IRS 1, first noted by Yusef-Zadeh et al. (1990) and discussed in more detail by Reipurth et al. (2013). As proposed by the latter authors, IRS 1 is quite likely an elliptical galaxy powering the radio galaxy. The nearby source IRS 2 (see Figure 1) also reported by Yusef-Zadeh et al. (1990) and Reipurth et al. (2013), has no associated radio emission at a 3- $\sigma$ upper limit of $69 \mu \mathrm{Jy}$.

Reipurth et al. (2013) did a detailed optical and near-IR continuum and line study of $\mathrm{HH} 222$ and found that it has proper motions of $107 \pm 25 \mathrm{~km} \mathrm{~s}^{-1}$ toward P.A. $=$ $329^{\circ} \pm 10^{\circ}$. These results pointed to a source to the SE of HH 222 as its exciting source. These authors proposed that the most likely source was V380 Ori $\left(=\mathrm{BD}-06^{\circ} 1253=\right.$ Parenago $2393=$ HBC $164=$ Haro 4-235), located at $22 ! 6$ to the SE of HH 222. V380 Ori is a hierarchical quadruple system composed of a Herbig Be star with an infrared companion separated by $0 !^{\prime \prime} 15$ toward a P.A. of $204^{\circ}$ that was discovered by Leinert et al. (1997). These two components were labeled as $\mathrm{Aa}$ and Ab, respectively, by Reipurth et al. (2013). Component Aa is itself a spectroscopic binary, with the Herbig Be star having an effective temperature of $10500 \pm 500 \mathrm{~K}$ and a luminosity of $\sim 200 L_{\odot}$ and the spectroscopic companion having an effective temperature of $5500 \pm 500 \mathrm{~K}$ and a luminosity of $\sim 3 L_{\odot}$ (Alecian et al. 2009). Here we will consider the spectroscopic binary as a single object. A fourth component of spectral type M5 or M6 (labeled B by Reipurth et al. 2013) is located at a distance of 8." 8 at a P.A. of $120^{\circ}$. 


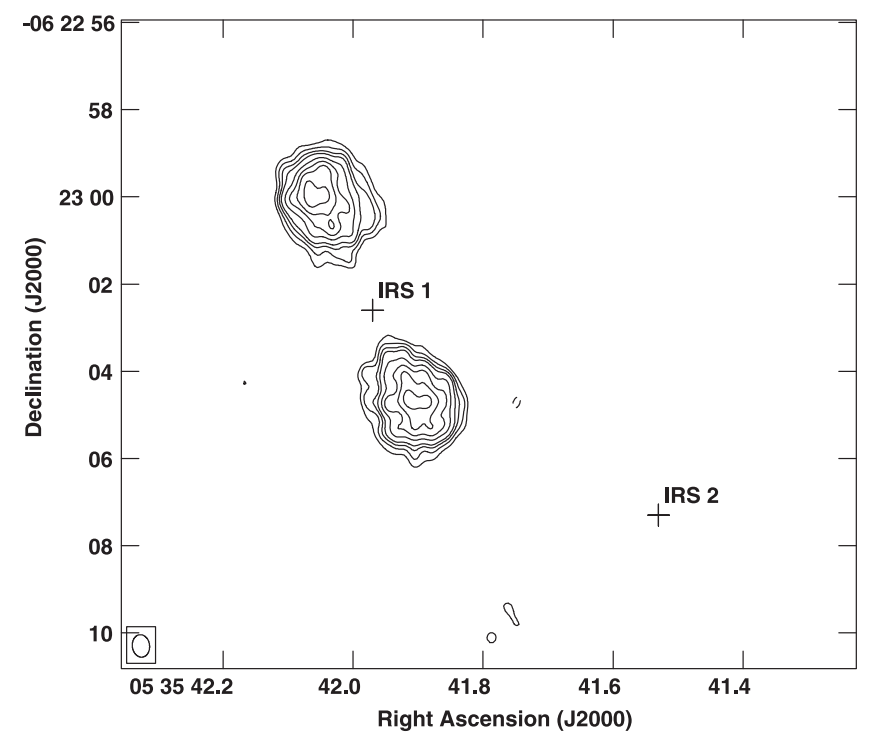

Figure 1. VLA $4.86 \mathrm{GHz}$ continuum contour image of the core of the HH 222 region, made from the 1991 and 2008 data discussed by Trejo \& Rodríguez (2010). The contours are $-4,-3,3,4,5,6,8,10,12$, and 15 times $23 \mu \mathrm{Jy}$ beam $^{-1}$, the rms noise of the image. The classic double-lobe morphology of a radio galaxy is evident. The half-power contour of the synthesized beam is shown in the bottom left corner $\left(0 . .52 \times 0\right.$. 40 ; PA $\left.=+7^{\circ}\right)$. The crosses mark the positions of the two infrared sources discussed by Yusef-Zadeh et al. (1990) and Reipurth et al. (2013).

Reipurth et al. (2013) also found a new Herbig-Haro object, $\mathrm{HH} 1041$, located at 17!3 to the SE of V380 Ori in the opposite direction of HH222 and likely forming part of a counterflow to $\mathrm{HH} 222$. Figure 14 of Reipurth et al. (2013) shows the positions of HH 222, V380 Ori, and HH 1041.

Since the exciting sources of $\mathrm{HH}$ objects are known to practically always be associated with faint free-free emission at centimeter wavelengths (e.g., Rodríguez \& Reipurth 1998), we did a search for such a source, concatenating several epochs of observation from the VLA archives to achieve the highest sensitivity possible and we also obtained new observations using the ultrasensitive Jansky VLA.

\section{OBSERVATIONS}

The VLA archive observations were made at C-band (4.86 $\mathrm{GHz}$ ) during 11 epochs that are summarized in Table 1. The average epoch of these data is 1998.01. These observations were made with the phase center at or very close to the position of HH $1 / 2$ VLA $1\left(\alpha(\mathrm{J} 2000)=05^{\mathrm{h}} 36^{\mathrm{m}} 22^{\mathrm{s}} .84 ; \delta(\mathrm{J} 2000)=\right.$ $\left.-06^{\circ} 46^{\prime} 06^{\prime \prime} 2\right)$, the exciting source of the HH $1 / 2$ system (Pravdo et al. 1985; Rodríguez et al. 2000). The data were calibrated following the standard procedures in the AIPS (Astronomical Image Processing System) software package of $\mathrm{NRAO}^{5}$ and concatenated in a single file. An image with weighting of ROBUST $=5$ (equivalent to natural weighting in AIPS; Briggs 1995) was made to optimize the sensitivity of the image at the expense of losing some angular resolution. A source was clearly detected in close vicinity $(\sim 0$ !! 1$)$ of the Herbig Be star V380 Ori (see Figure 2). Since the observations were made with bandwidths of $50 \mathrm{MHz}$ (two of them, adjacent in frequency) and V380 Ori is located about $3 ! 2$ to the NE of

\footnotetext{
5 The National Radio Astronomy Observatory is a facility of the National Science Foundation operated under cooperative agreement by Associated Universities, Inc.
}

HH 1-2 VLA1, the radio source presents significant bandwidth smearing. Under these limitations, the source appears to be spatially unresolved $(\leqslant 2$ !' 0$)$. Its total flux density is $0.20 \pm 0.03 \mathrm{mJy}$. All flux densities presented here have been corrected for the primary beam response.

The new observations were made with the Karl G. Jansky Very Large Array of NRAO in the C (4.4-6.4 GHz) and X (7.9-9.9 GHz) bands during 2012 May 26, under project 12A240. At that time the array was in its B configuration. The phase center was at $\alpha(2000)=05^{\mathrm{h}} 36^{\mathrm{m}} 22^{\mathrm{s}} .00 ; \delta(2000)=$ $-06^{\circ} 46^{\prime} 07$ !. 0 . The absolute amplitude calibrator was $0137+331$ and the phase calibrator was J0541-0541.

The digital correlator of the JVLA was configured at each band in 16 spectral windows of $128 \mathrm{MHz}$ width, each subdivided into 64 channels of $2 \mathrm{MHz}$. The narrow width of each channel eliminates the bandwidth smearing that limited observations away from the phase center in the classic VLA. The total bandwidth of the observations was about $2.048 \mathrm{GHz}$ in a full-polarization mode. The data were analyzed in the standard manner using the CASA (Common Astronomy Software Applications) package of NRAO, although for some stages of the analysis we used the AIPS package. For all of the imaging, we used the ROBUST parameter of CLEAN set to two (equivalent to natural weighting in CASA) to obtain a better sensitivity.

We confirm the detection of the radio source in close vicinity to V380 Ori. Its total flux densities were $0.14 \pm 0.03 \mathrm{mJy}$ and $0.25 \pm 0.04 \mathrm{mJy}$, for the $\mathrm{C}$ and $\mathrm{X}$ bands, respectively. Assuming a power law for the emission, the radio flux density of V380 Ori for epoch 2012 May 26 can be described as

$$
\left[\frac{S_{\nu}}{\mathrm{mJy}}\right]=0.14 \pm 0.03\left[\frac{\nu}{5.4 \mathrm{GHz}}\right]^{1.2 \pm 0.5} .
$$

An image of the radio source was made combining the $\mathrm{C}$ and $\mathrm{X}$ band data, covering an almost continuous frequency range from 4.4 to $9.9 \mathrm{GHz}$. The image is shown in Figure 3. We searched unsuccessfully for additional sources in the region. In particular, we did not detect radio emission from component $\mathrm{B}$ at a 3- $\sigma$ level of $34 \mu \mathrm{Jy}$.

\section{THE NATURE OF THE RADIO SOURCE DETECTED IN CLOSE VICINITY TO V380 ORI}

\subsection{A Radio Jet}

Several characteristics of the radio source favor an interpretation in terms of a thermal radio jet (Anglada 1996; Rodríguez 1997; Eisloffel et al. 2000, p. 815; Anglada et al. 2015):

i. The spectral index of $1.2 \pm 0.5$ is consistent with the partially optically thick free-free emission produced by thermal jets.

ii. The source exhibits little or no temporal flux density variation, as generally observed in this type of sources. Most of the radio jets that have been monitored over the years show no evidence of variability above the $10 \%$ 20\% level (e.g., Rodríguez et al. 2008, 2014; Loinard et al. 2010; Carrasco-González et al. 2012).

iii. The source in the combined $\mathrm{C}$ and $\mathrm{X}$ band image has deconvolved dimensions of 1 !" $3 \pm 0$ !. $4 \times 0$ !" $8 \pm 0$ !. 3 ; $\mathrm{PA}=+161^{\circ} \pm 36^{\circ}$, with the position angle, modulo $180^{\circ}$, consistent with the position angle of the proper 
Table 1

Parameters of VLA Archive Observations in C-band

\begin{tabular}{|c|c|c|c|c|c|c|}
\hline \multirow{2}{*}{$\begin{array}{l}\text { Epoch } \\
(\mathrm{yy} / \mathrm{mm} / \mathrm{dd})\end{array}$} & \multirow[b]{2}{*}{ Project } & \multirow[b]{2}{*}{ Configuration } & \multicolumn{2}{|c|}{ Phase Center } & \multirow{2}{*}{$\begin{array}{l}\text { Amplitude } \\
\text { Calibrator }\end{array}$} & \multirow{2}{*}{$\begin{array}{c}\text { Phase } \\
\text { Calibrator }\end{array}$} \\
\hline & & & $\alpha(\mathrm{J} 2000)$ & $\delta(\mathrm{J} 2000)$ & & \\
\hline 1984 Oct 02 & AR114 & $\mathrm{D}$ & $05^{\mathrm{h}} 36^{\mathrm{m}} 23^{\mathrm{s}} .865$ & $-06^{\circ} 45^{\prime} 11^{\prime \prime} 66$ & $3 \mathrm{C} 286$ & 0539-057 \\
\hline 1985 Nov 21 & AR131 & $\mathrm{D}$ & $05^{\mathrm{h}} 36^{\mathrm{m}} 22^{\mathrm{s}} .846$ & $-06^{\circ} 46^{\prime} 08$. $^{\prime \prime} 59$ & $3 \mathrm{C} 286$ & 0539-057 \\
\hline 1986 Jan 13 & AR131 & $\mathrm{D}$ & $05^{\mathrm{h}} 36^{\mathrm{m}} 22.846$ & $-06^{\circ} 46^{\prime} 08^{\prime \prime} .59$ & $3 \mathrm{C} 286$ & 0539-057 \\
\hline 1986 Mar 01 & AR131 & A & $05^{\mathrm{h}} 36^{\mathrm{m}} 22^{\mathrm{s}} .846$ & $-06^{\circ} 46^{\prime} 08^{\prime \prime} .59$ & $3 \mathrm{C} 286$ & 0539-057 \\
\hline 1986 Mar 03 & AR131 & A & $05^{\mathrm{h}} 36^{\mathrm{m}} 22^{\mathrm{s}} .846$ & $-06^{\circ} 46^{\prime} 08^{\prime \prime} .59$ & $3 \mathrm{C} 286$ & 0539-057 \\
\hline 1986 Sep 12 & AR131 & A & $05^{\mathrm{h}} 36^{\mathrm{m}} 22^{\mathrm{s}} .846$ & $-06^{\circ} 46^{\prime} 08^{\prime \prime} .59$ & $3 \mathrm{C} 286$ & 0539-057 \\
\hline 1986 Sep 13 & AR131 & A & $05^{\mathrm{h}} 36^{\mathrm{m}} 22^{\mathrm{s}} .846$ & $-06^{\circ} 46^{\prime} 08^{\prime \prime} .59$ & $3 \mathrm{C} 286$ & 0539-057 \\
\hline 1986 Dec 09 & AR131 & $\mathrm{C}$ & $05^{\mathrm{h}} 36^{\mathrm{m}} 22^{\mathrm{s}} .846$ & $-06^{\circ} 46^{\prime} 08^{\prime \prime} 59$ & $3 \mathrm{C} 286$ & 0539-057 \\
\hline 1992 Nov 02 & AR278 & A & $05^{\mathrm{h}} 36^{\mathrm{m}} 22^{\mathrm{s}} .846$ & $-06^{\circ} 46^{\prime} 08^{\prime \prime} .59$ & $3 \mathrm{C} 286$ & 0539-057 \\
\hline 1992 Dec 18 & AR278 & A & $05^{\mathrm{h}} 36^{\mathrm{m}} 22^{\mathrm{s}} .846$ & $-06^{\circ} 46^{\prime} 08^{\prime \prime} .59$ & $3 \mathrm{C} 286$ & 0539-057 \\
\hline 1992 Dec 19 & AR278 & A & $05^{\mathrm{h}} 36^{\mathrm{m}} 22^{\mathrm{s}} .846$ & $-06^{\circ} 46^{\prime} 08^{\prime \prime} 59$ & $3 \mathrm{C} 286$ & 0539-057 \\
\hline
\end{tabular}

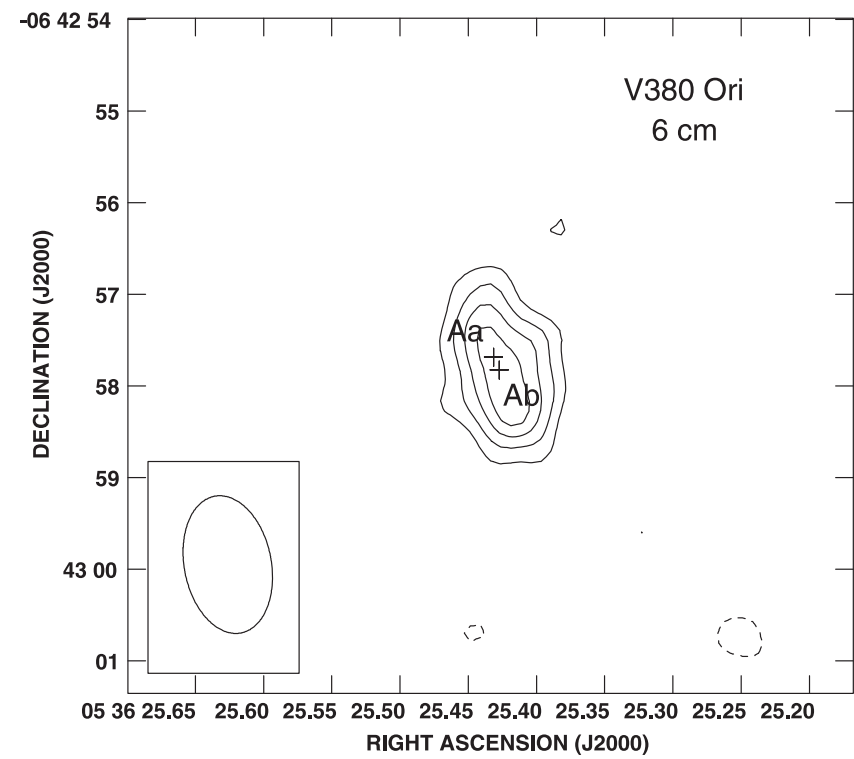

Figure 2. VLA $4.86 \mathrm{GHz}$ continuum contour image of the V380 Ori region, made from the archive data summarized in Table 1 . The contours are $-4,-3,3,4,5$, and 6 times $30.7 \mu \mathrm{Jy}_{\text {beam }}{ }^{-1}$. The image has been corrected for the response of the primary beam. The half-power contour of the synthesized beam has been corrected to include the effect of bandwidth smearing and is shown in the bottom left corner $\left(1 / .52 \times 0\right.$ ". 95 ; PA $\left.=+11^{\circ}\right)$. In this image the source appears unresolved and with a total flux density of $0.20 \pm 0.03 \mathrm{mJy}$. The positional accuracy of the VLA image is 0 !! 3 . The crosses mark the position of the Herbig Be star (component Aa) from van Leeuwen (2007) and from the infrared companion (component Ab) as derived from the offsets of Leinert et al. (1997).

motions of HH $222\left(329^{\circ} \pm 10^{\circ}\right.$; Reipurth et al. 2013) and the direction from V380 Ori to the core of HH 222 $\left(\simeq 331^{\circ}\right)$. We propose that this radio source traces the origin of the HH system.

\subsection{Is the Radio Source Associated with the Herbig Be Star in V380 Ori or with Its Infrared Companion?}

Our radio images show that the peak of the radio emission falls within $\sim 0$ !' 1 of the optical position of the Herbig Ae star as given by van Leeuwen (2007). Unfortunately, the quality of the available astrometry is insufficient to establish if the radio jet is associated with the Herbig Be star (Aa) or with its close infrared companion $(\mathrm{Ab})$. The primary star (Aa) hosts a dipole magnetic field with polar strength of $\sim 2 \mathrm{kG}$ (Alecian et al. 2009), suggesting the possibility of gyrosynchrotron

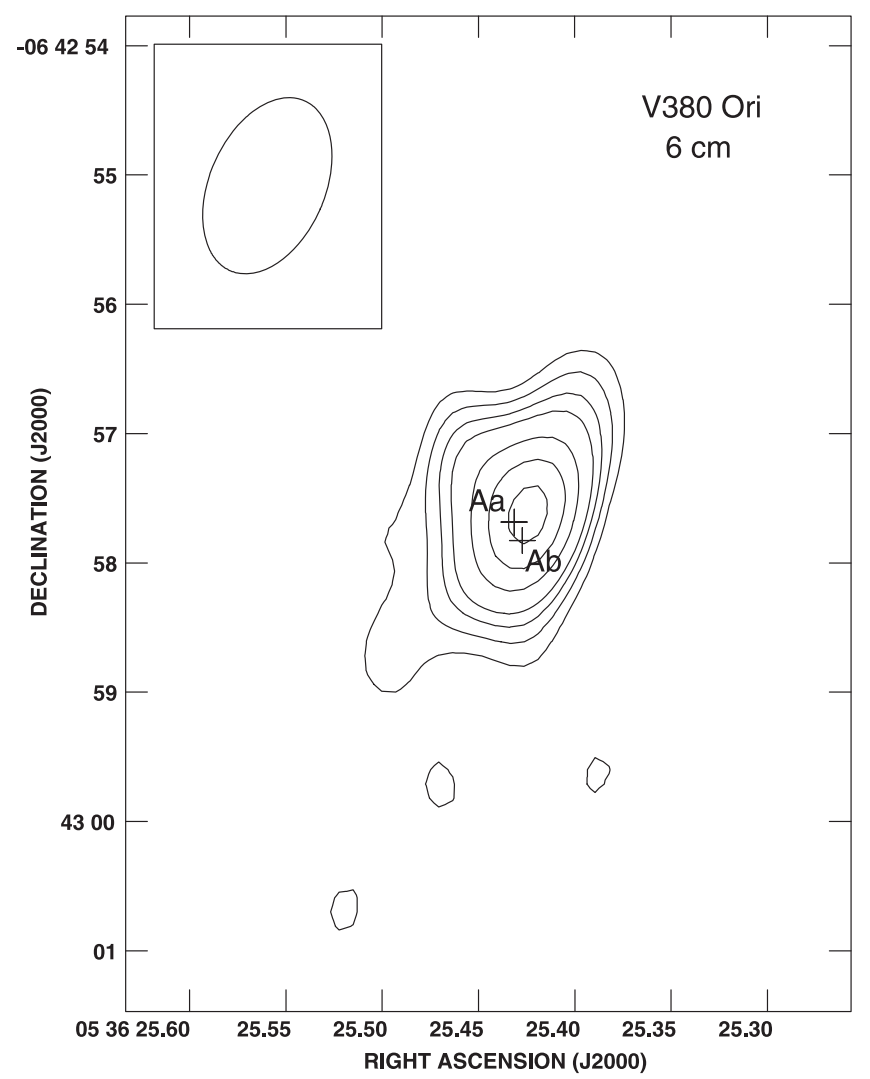

Figure 3. JVLA $6 \mathrm{~cm}$ continuum contour image of the V380 Ori region, made from the new (2012) Jansky VLA observations. The contours are $-4,-3,3,4$, $5,6,8,10$, and 12 times $11.2 \mu \mathrm{Jy}_{\text {beam }^{-1}}$, the rms noise of this region of the image. The image has been corrected for the response of the primary beam. The half-power contour of the synthesized beam is shown in the bottom left corner $\left(1^{\prime \prime} 43 \times 0\right.$ " $\left.89 ; \mathrm{PA}=-24^{\circ}\right)$. In this image the source appears marginally resolved with deconvolved dimensions of 1 !" $3 \pm 0$ !" $4 \times 0$ !. $8 \pm 0$ !" 3 ; $\mathrm{PA}=+161^{\circ} \pm 36^{\circ}$ and with a total flux density of $0.22 \pm 0.03 \mathrm{mJy}$. The positional accuracy of the VLA image is 0 " 1 . The crosses mark the position of the Herbig Be star (component Aa) from van Leeuwen (2007) and from the infrared companion (component $\mathrm{Ab}$ ) as derived from the offsets of Leinert et al. (1997).

emission from this star. However, the characteristics of the observed radio emission favor a free-free nature. Leinert et al. (1997) estimate a luminosity of the order of $170-180 L_{\odot}$ for component Aa and of 30-70 $L_{\odot}$ for component $\mathrm{Ab}$.

Levreault (1988) detected a redshifted molecular outflow associated with V380 Ori. With the evidence that a jet is present there, a new higher angular resolution and sensitivity 
molecular mapping of the region may provide valuable new information.

\subsection{The Radio Luminosity of V380 Ori}

The radio luminosity of radio jets, $S_{\nu} d^{2}$, where $S_{\nu}$ is the flux density at $8 \mathrm{GHz}$ in mJy and $d$ is the distance to the source in $\mathrm{kpc}$, is correlated with the bolometric luminosity of the source, $L_{\text {bol }}$ (Anglada et al. 2015), by

$$
\left(\frac{S_{\nu} d^{2}}{\mathrm{mJy} \mathrm{kpc}^{2}}\right)=0.008\left(\frac{L_{\mathrm{bol}}}{L_{\odot}}\right)^{0.6} \text {. }
$$

We first discuss the possibility that the radio jet is associated with component $\mathrm{Aa}$ of V380 Ori, that has a bolometric luminosity of $\sim 175 L_{\odot}$ (Leinert et al. 1997). Assuming that this source is located at a distance of $460 \mathrm{pc}$ (Reipurth et al. 2013), we expect a flux density of $\sim 0.84 \mathrm{mJy}$ at $\sim 8 \mathrm{GHz}$ from the correlation given above. This is a factor of 3.8 larger than the measured value of $0.22 \mathrm{mJy}$, indicating that this object is underluminous in the radio. This probably results from the fact that, with an age of about 2 million years (Alecian et al. 2009), component Aa of V380 Ori is a more evolved object than those used to establish the correlation (that typically have ages below a few times $10^{5}$ year, Anglada et al. 2015). This correlation most likely reflects the fact that in very young stars the luminosity is dominated by accretion, which in turn is correlated with the outflow activity that is traced by the radio free-free emission. In these very young objects the stellar contribution to the bolometric luminosity is relatively unimportant. As the star evolves the accretion decreases and the relative stellar contribution becomes more important, making the star radio-underluminous with respect to the Anglada et al. correlation. A similar underluminous radio jet has been recently found in AB Aur (Rodríguez et al. 2014). In contrast, the nearby radio jet $\mathrm{HH} 1-2$ VLA1 is a much younger class 0 protostar (age $<10^{5}$ year; Andre et al. 2000, p. 59) and has a flux density of $\sim 1.0 \mathrm{mJy}$ at $\sim 8 \mathrm{GHz}$ (Rodríguez et al. 2000). With a bolometric luminosity of $23 L_{\odot}$ (Fischer et al. 2010), this source is about four times overluminous in the radio with respect to the correlation.

On the other hand, if we assume that the jet comes from component $\mathrm{Ab}$ (the infrared companion) that has a luminosity of $\sim 50 L_{\odot}$, the expected flux density would be $\sim 0.40 \mathrm{mJy}$ at $\sim 8 \mathrm{GHz}$. This is only a factor of two larger than the measured value of $0.22 \mathrm{mJy}$. However, in this case the Herbig Be star (component Aa) would be strongly underluminous in the radio, suggesting considerable evolution. In any case, the data from which the correlation is derived shows considerable scatter, and better and more abundant data are needed to clearly establish observationally if there is, as expected, a correlation between radio luminosity and age for young stars. The search for this correlation is complicated, as in this case, by the multiplicity of young stellar systems.

\section{CONCLUSIONS}

We present the analysis of archive VLA data as well as new high sensitivity Jansky VLA observations toward V380 Ori. The main results of our study can be summarized as follows.
1. We detect a radio counterpart to V380 Ori that has spectral index, morphology, and lack of time variability all consistent with the source being a free-free jet.

2. The major axis of the radio source aligns, within the observational error, with the position angle of the proper motions of HH 222 and the direction from V380 Ori to the core of HH 222. We propose that this radio jet traces the origin of the large scale $\mathrm{HH}$ outflow, supporting the suggestion of Reipurth et al. (2013) that V380 Ori is the exciting source of the giant HH 222/HH 1041 system.

3 . We cannot establish unambiguously if the jet originates from the Herbig Be star (component Aa) or from its close (separation of 0 !' 15) infrared companion (component Ab). In any case, the radio luminosity of the jet is smaller than expected from the bolometric luminosity of either component. We suggest that better and more abundant data are needed to clearly establish if there is a decrease in thermal radio luminosity with age for young stars.

We thank an anonymous referee for valuable comments. This research has made use of the SIMBAD database, operated at CDS, Strasbourg, France. L.F.R. is grateful to CONACyT, Mexico and DGAPA, UNAM for their financial support. G.A. acknowledges support from MINECO (Spain) grant AYA2014-57369-C3-3-P (co-funded with FEDER funds).

\section{REFERENCES}

Alecian, E., Wade, G. A., Catala, C., et al. 2009, MNRAS, 400, 354

Andre, P., Ward-Thompson, D., \& Barsony, M. 2000, Protostars and Planets IV (Tucson, AZ: Univ. Arizona Press)

Anglada, G. 1996, in ASP Conf. Ser. 93, Radio Emission from the Stars and the Sun, ed. A. R. Taylor \& J. M. Paredes (San Francisco, CA: ASP), 3

Anglada, G., Rodríguez, L. F., \& Carrasco-Gonzalez, C. 2015, in Proc. of Advancing Astrophysics with the Square Kilometre Array (AASKA14), 121 Briggs, D. 1995, PhD thesis, New Mexico Inst. of Mining and Technology

Carrasco-González, C., Osorio, M., Anglada, G., et al. 2012, ApJ, 746, 71

Castets, A., Reipurth, B., \& Loinard, L. 2004, A\&A, 427, 895

Cohen, M., \& Schwartz, R. D. 1983, ApJ, 265, 877

Eisloffel, J., Mundt, R., Ray, T. P., \& Rodriguez, L. F. 2000, Protostars and Planets IV (Tucson, AZ: Univ. Arizona Press)

Fischer, W. J., Megeath, S. T., Ali, B., et al. 2010, A\&A, 518, L122

Leinert, C., Richichi, A., \& Haas, M. 1997, A\&A, 318, 472

Levreault, R. M. 1988, ApJS, 67, 283

Loinard, L., Rodríguez, L. F., Gómez, L., et al. 2010, RMxAA, 46, 375

Morgan, J. A., Snell, R. L., \& Strom, K. M. 1990, ApJ, 362, 274

Pravdo, S. H., Rodriguez, L. F., Curiel, S., et al. 1985, ApJL, 293, L35

Reipurth, B., Bally, J., Aspin, C., et al. 2013, AJ, 146, 118

Reipurth, B., Chini, R., Krugel, E., Kreysa, E., \& Sievers, A. 1993, A\&A, 273, 221

Reipurth, B., \& Sandell, G. 1985, A\&A, 150, 307

Rodriguez, L. F. 1997, in IAU Symp. 182, Herbig-Haro Flows and the Birth of Stars (Dordrecht: Kluwer), 83

Rodríguez, L. F., Delgado-Arellano, V. G., Gómez, Y., et al. 2000, AJ, 119,882

Rodríguez, L. F., Moran, J. M., Franco-Hernández, R., et al. 2008, AJ, 135,2370

Rodríguez, L. F., \& Reipurth, B. 1998, RMxAA, 34, 13

Rodríguez, L. F., Zapata, L. A., Dzib, S. A., et al. 2014, ApJL, 793, L21

Trejo, A., \& Rodríguez, L. F. 2010, RMxAA, 46, 357

van Leeuwen, F. 2007, A\&A, 474, 653

Yusef-Zadeh, F., Cornwell, T. J., Reipurth, B., \& Roth, M. 1990, ApJL, 348, L61 